\title{
The Effect of Smoke Combined with the Multicancer Marker, Rs6983267, Located at Region 3 of Chromosome 8q24 in Prostate Cancer Patients
}

Amalia Papanikolopoulou ${ }^{1}$, Olfert Landt ${ }^{2}$, Martin Reczko ${ }^{3}$, Konstantinos Ntoumas ${ }^{4}$, Stefanos Bolomitis ${ }^{4}$, Stavros I Tyritzis ${ }^{5}$, Constantinos Konstantinides ${ }^{5}$ and Nikolaos Drakoulis ${ }^{1 *}$

${ }^{1}$ Department of Pharmaceutical Technology, School of Pharmacy, National and Kapodistrian University of Athens, Greece ${ }^{2}$ TIB MOLBIOL, Berlin, Germany

${ }^{3}$ Biomedical Sciences Research Center, Alexander Fleming, Vari, Greece

${ }^{4}$ Departement of Urology, General Hospital Gennimatas, Athens, Greece

${ }^{5}$ Department of Urology, General Hospital Laiko, Medical School, National and Kapodistrian University of Athens, Greece

\begin{abstract}
Introduction: Common variants on human chromosome $8 \mathrm{q} 24$ were found to be associated with prostate cancer risk with different frequency and incidence among the investigated populations. We examined the effect of smoke on this type of cancer and its relationship with the risk variant rs6983267, located at region 3 of chromosome 8q24, in a prostate cancer case-control study conducted in the Greek population in light, intermediate and heavy smokers.

Materials and methods: Samples of total blood from 74 patients with histologically confirmed prostate cancer and 24 healthy individuals were genotyped using real time polymerase chain reaction (PCR). Tumor-node-metastasis (TNM) stage, Gleason score and levels of prostate-specific antigen (PSA) at diagnosis were included in the analysis.

Results: Light (Packyears, PY<10) and heavy $(P Y>30)$ smokers are positive associated with prostate cancer, with an additive risk for the carriers of rs6983267 with positive smoking history (ORadj=21.36, C.L=3.79-120.39) to develop the disease.

Conclusions: The SNP, rs6983267, has an independent risk for carriers to develop prostate cancer and in combination with smoke; it confers additive risk for the disease, similarly to others, well established risk factors such as age, family history and ethnicity.
\end{abstract}

Keywords: Prostate cancer; Smoke; 8q24; Rs6983267

Abbreviations: SNP: Simple Nucleotide Polymorphism; PSA: Prostate Specific Antigen; DRE-Digital Rectal Examination; PCR: Polymerase Chain Reaction; GWAS: Genome-Wide Association Studies; PAR: Population Attributable Risk; UADT: Upper AeroDigestive Tract; FSH: Follicle Stimulating Hormone; IARC: International Agency for Research on Cancer; ROS: Reactive Oxygen Species; tPA: Tissue-type Plasminogen Activator; MMPs: Metalloproteinases

\section{Introduction}

The evidence for a genetic susceptibility to prostate cancer (PCa) has been well documented but the only firmly established risk factors for PCa are age, family history and ethnicity [1]. In an attempt to identify genetic variants underlying risk for PCa, genome-wide linkage and association studies (GWAS) have been performed and multiple chromosomal regions have been designated to harbour major susceptibility genes for PCa [2]. Three independent regions on chromosome 8q24 have been investigated thoroughly, at the beginning in small populations (Islander) and afterwards in bigger races like African-American, European-American, Japanese-American, Natives of Hawaii and Latinos, with different frequency and indecent for the PCa [3-6]. In men of European ancestry the single nucleotide polymorphism (SNP) rs6983267, at the region 3 of chromosome 8q24 has shown the highest odds ratio and population attributable risk (PAR) for PCa, compared to other SNPs at the same region [3]. Rs6983267 also has been associated with colorectal cancer, kidney and thyroid cancers and smoking-related cancers and thus was characterised as a multicancer marker with a genetic role yet to be clarified [7-9].

Smoke is a well established environmental carcinogen and it has been associated with many malignancies including the urinary track: bladder, prostate and kidney [10]. For PCa there are conflicting data on the effect of cigarette smoking on serum levels of the various sex hormones and epidemiological studies have not compounded to a conclusive relationship. Smoke-mediated changes have been documented not only in endocrine pathways but also in enzymatic systems showing positive association between current smoking and fatal cancer of the prostate in many prospective cohort studies [11].

\section{Methods}

\section{Study subjects}

In total, 74 patients with $\mathrm{PCa}$ and positive smoking history participated in the study. They were identified and recruited from the "Laiko" General University Hospital and from the "Gennimatas" General Hospital with an age distribution between $48-87$ years. The inclusion criterion for case subjects was histological biopsy-confirmed adenocarcinoma of the PCa, diagnosed between October 2008 and January 2010. Tumor-node-metastasis (TNM) stage, Gleason score as

*Corresponding author: Nikolaos Drakoulis, Department of Pharmaceutical Technology, School of Pharmacy, National and Kapodistrian University of Athens, Greece, Tel: +302109948200; Fax: +302109948209; E-mail: drakoulis@pharm.uoa.gr

Received December 06, 2011; Accepted January 25, 2012; Published January 27, 2012

Citation: Papanikolopoulou A, Landt O, Reczko M, Ntoumas K, Bolomitis S, et al (2012) The Effect of Smoke Combined with the Multicancer Marker, Rs6983267. Located at Region 3 of Chromosome $8 \mathrm{q} 24$ in Prostate Cancer Patients. Metabolomics 2:106. doi:10.4172/2153-0769.1000106

Copyright: (C) 2012 Papanikolopoulou A, et al. This is an open-access article distributed under the terms of the Creative Commons Attribution License, which permits unrestricted use, distribution, and reproduction in any medium, provided the original author and source are credited. 
determined by biopsy and levels of prostate-specific antigen (PSA) at diagnosis were available for all the patients.

Twenty four control subjects with positive smoking history were recruited concurrently with case subjects, randomly selected from the «KAT» General Hospital of Attika with an age distribution between 47-71 years. They were all healthy individuals, with PSA levels $<4 \mathrm{ng} /$ $\mathrm{ml}$, negative Digital Rectal Examination (DRE) and no family history.

Both case and control subjects provided written informed of consent. The study received institutional approval from the scientific committee (Reference Number 697/17-10-2008).

\section{Genotyping of SNP rs6983267}

Whole EDTA blood samples $(5 \mathrm{ml})$ were drawn from all subjects and DNA was extracted using the Wizard Genomic DNA Purification Kit (Promega, USA). The yield was measured by spectrophotometry (NanoDrop, 1000, UK). Genotyping was performed by Real-time PCR using the Light Cycler 480 system (Roche Diagnostics, Germany) using the primers rs6983267 F: 5'-CTATCCATAAAACAgAgggACgAAT and rs6983267 R: 5' - gggTTCCTgCCCTTTgATT and the hybridization probes Sensor wt: 5'-TTTCTCAgTgCCTTTCATCTgC-FL and anchor LC640-gCTCAAAggACgATgAgggTATTAACTCTg-PH, Fast Start hybridization probe master (Roche) running 45 cycles each 5 $\sec 95^{\circ} \mathrm{C}, 10 \sec 60^{\circ} \mathrm{C}$ and $10 \sec 72^{\circ} \mathrm{C}$ and a subsequent melting curve from $45^{\circ} \mathrm{C}$ to $85^{\circ} \mathrm{C}$ with a slope $0.2^{\circ} \mathrm{C} / \mathrm{sec}$ and continuous fluorescence detection.

\section{Statistical analysis}

Qualitative variables are presented with absolute and relative frequencies, while quantitative variables are presented with mean (standard deviation). For the comparison of mean values between two groups, student's t-test was used. For the comparison of proportions between the $\mathrm{PCa}$ and the control groups chi-square tests were computed. Logistic regression analysis was performed in order to evaluate the association of pack years with the presence of PCa. Adjusted odds ratios with 95\% confidence intervals were performed from the results of logistic regression analysis. All reported $\mathrm{p}$ values are two-tailed. Statistical significance was set at $\mathrm{p}<0.05$ and analyses were conducted using SPSS statistical software (version 17.0) (Figures 1-3).

\section{Results}

Data from 74 patients with PCa and 24 controls were analyzed. Sample characteristics of the PCa and control groups are presented in Table 1. Significantly greater mean age was found in the PCa group (67.5 \pm 6.6 vs. $56.6 \pm 9.4)$. The overall distribution of $\mathrm{rs} 6983267$ genotype TT (wild type), GT (heterozygotes), GG (homozygotes) was $18.9 \%, 54.1 \%, 27.0 \%$ in patients and $66.7 \%, 29.2 \%, 4.2 \%$ in controls, respectively. The proportion of homozygotes (GG) or heterozygotes (GT) was significantly greater for PCa group $(\mathrm{p}<0.001)$. Also, the proportion of subjects with pack years less than ten or more than thirty was significantly greater in the PCa group. When multiple analysis was conducted, (Table 2) it was found that after adjusting for age and rs6983267 genotype the pack years were independently associated with PCa. Specifically, it was found that subjects with less than ten pack years had 11.49 times greater likelihood for PCa $(\mathrm{p}=0.016)$ compared to those with ten to thirty pack years. Furthermore, subjects with more than thirty pack years had 10.88 times greater odds for $\mathrm{PCa}(\mathrm{p}=0.014)$ compared to those with ten to thirty pack years. Additionally, the odds for PCa adjusted for age and smoke was 21.21 times greater for homozygotes or heterozygotes of rs6983267 genotype ( $\mathrm{p}=0.001)$. The

\section{Pack Years and incidence of $\mathrm{PCa}$}

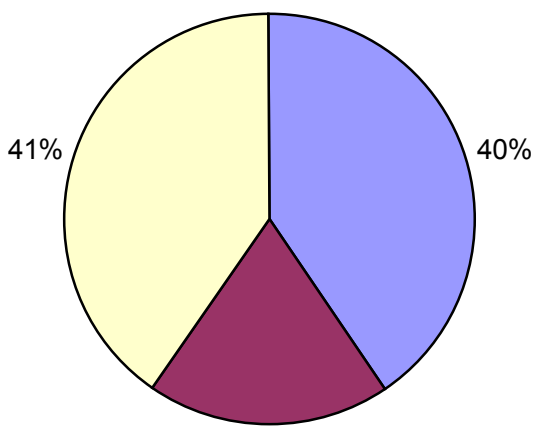

$19 \%$

Figure 1: Pack Years and incidence of PCa.

\section{Genotype and incidence of PCa}
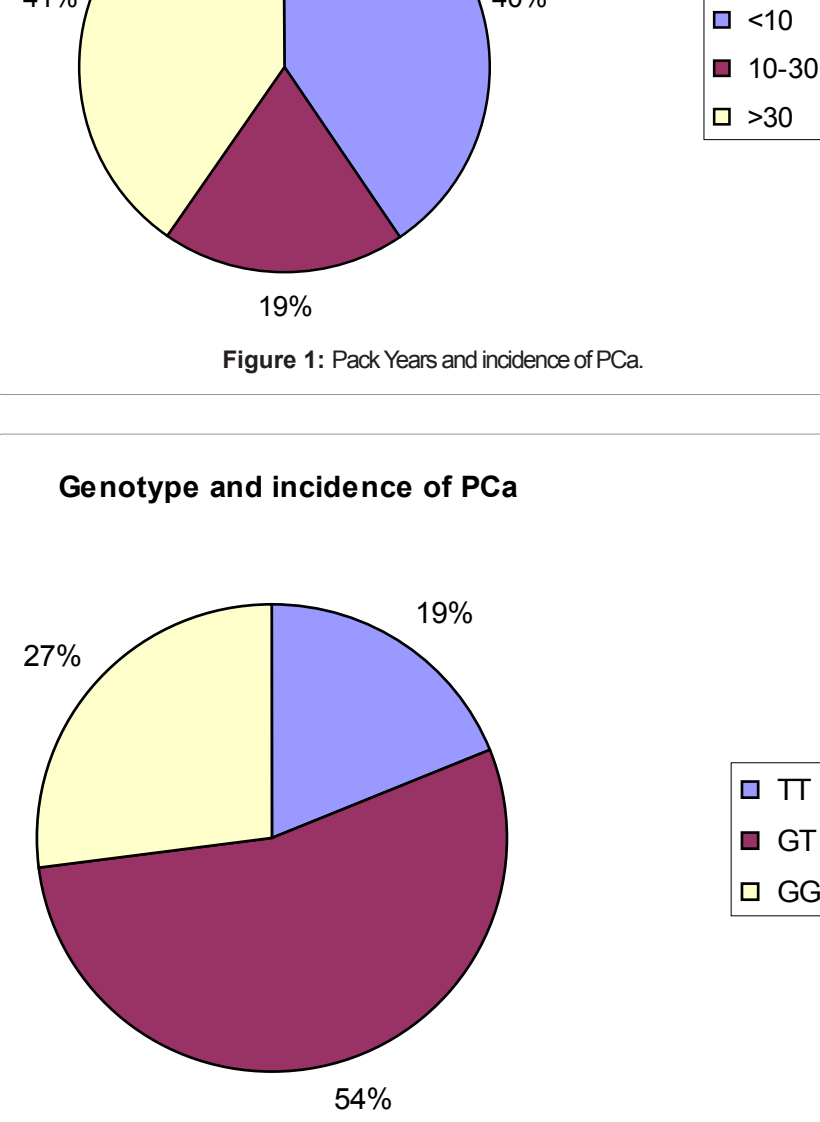

Figure 2: Genotype and incidence of PCa.

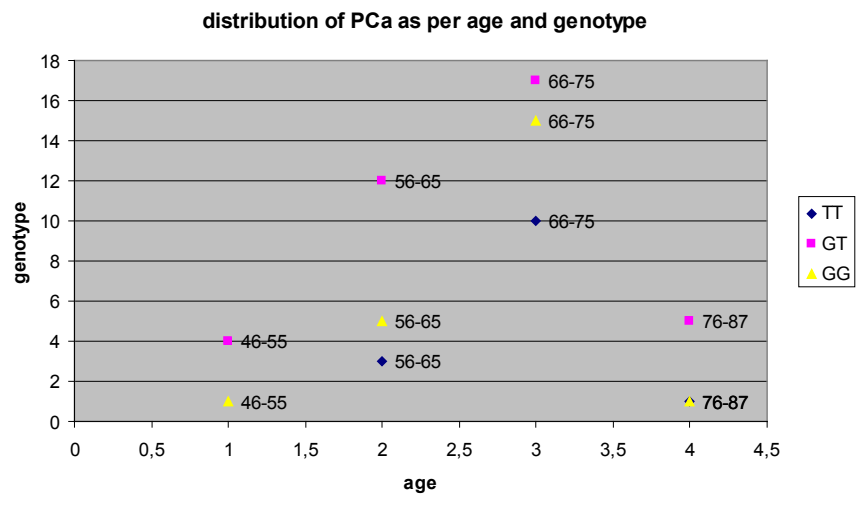

Figure 3: Distribution of PCa as per age and genotype.

proportion of homozygotes or heterozygotes for rs6983267 genotype for those with less than ten, ten to thirty and more than thirty pack years was $69.7 \%, 62.5 \%$ and $75.8 \%$ respectively $(\mathrm{p}=0.510)$. 


\begin{tabular}{|l|l|l|l|}
\hline & Control & Cases & $\mathrm{P}$ \\
\hline & $\mathrm{N}(\%)$ & $\mathrm{N}(\%)$ & $\mathrm{X}^{2}$ test \\
\hline Age (years), mean (SD) & $56.6(9.4)$ & $67.5(6.6)$ & $<0.001^{*}$ \\
\hline Pack years & & & \\
\hline$<10$ & $3(12.5)$ & $30(40.5)$ & $<0.001^{*}$ \\
\hline $10-30$ & $18(75)$ & $14(18.9)$ & \\
\hline$>30$ & $3(12.5)$ & $30(40.5)$ & \\
\hline RT-PCR & & & \\
\hline TT & $16(66.7)$ & $14(18.9)$ & $<0.001$ \\
\hline GT & $7(29.2)$ & $40(54.1)$ & \\
\hline GG & $1(4.2)$ & $20(27.0)$ & \\
\hline RT-PCR & & & \\
\hline GT/GG & $8(33.3)$ & $60(81.1)$ & $<0.001$ \\
\hline
\end{tabular}

*Student's t-test

Table 1: Characteristics of the prostate cancer and control group.

\begin{tabular}{|l|l|l|}
\hline & $\mathrm{OR}(95 \% \mathrm{Cl})$ & $\mathrm{P}$ \\
\hline Age (years) & $1.14(1.04-1.26)$ & 0.006 \\
\hline Pack years & & \\
\hline $10-30$ & $1.00 \ddagger$ & \\
\hline$<10$ & $11.49(1.56-84.52)$ & 0.016 \\
\hline$>30$ & $10.88(1.63-72.5)$ & 0.014 \\
\hline RT-PCR & & \\
\hline TT & 1.00 & \\
\hline GT/GG & $21.21(3.75-119.92)$ & 0.001 \\
\hline
\end{tabular}

łindicates reference category

Table 2: Odds ratios (OR) and $95 \%$ confidence intervals $(\mathrm{CI})$ derived from multiple logistic regression analysis with dependent variable the presence of prostate cancer.

\section{Discussion}

In men with European ancestry, rs6983267, has shown the highest odds ratio and population attributable risk \% (PAR\%) for $\mathrm{PCa}$, compared to other SNPs at the same region with little evidence of linkage disequilibrium with them and an overall population frequency in northern Europeans of $50 \%$ for the at risk allele [3]. When we examined rs6983267 in the Greek population, in a PCa case-control study, homozygotes or heterozygotes had 2.84 times greater likelihood for $\mathrm{PCa}(\mathrm{p}-0.002)$ and the overall population frequency for the $\mathrm{G}$ allele was $61.85 \%$. The carriers had almost two times greater odds for having the $\mathrm{G}$ allele ( $\mathrm{p}=0.001$ ) with a sensitivity for the disease of $81.40 \%$ [12].

Rs6983267 has also been associated with colorectal cancer, kidney, thyroid cancers and smoking-related cancers and so was characterised as a multicancer marker [7-9] with an important carcinogenetic role yet to be clarified. In the study of Lani Park et al. [9] note worthy are the associations between rs6983267 and the upper aero-digestive tract (UADT) cancers (ORadj $=1.69,95 \% \mathrm{CI}=1.28,2.24$ ), particularly in oropharynx (ORadj $=1.80,95 \% \mathrm{CI}=1.30,2.49)$ and larynx $($ ORadj $=2.04$, $95 \% \mathrm{CI}=1.12,3.72)$. When they stratified the analysis by smoking status, rs6983267 was positively associated with lung cancer among eversmokers (ORadj $=1.45,95 \% \mathrm{CI}=1.05,2.00$ ) and inversely associated with bladder cancer among ever-smokers (ORadj $=0.35,95 \% \mathrm{CI}=0.14$, 0.83 ). The positive association with UADT cancers, independent of tobacco smoking, indicates that rs6983267 may play a role in nonsmoking related pathways for these cancers. The positive association with lung cancer among ever-smokers indicates that rs6983267 may also play an important role in tobacco related carcinogenesis involving target specific carcinogens and perhaps metabolic, DNA repair and other related pathways. In our study, light $(\mathbf{P Y}<\mathbf{1 0})$ and heavy $(\mathbf{P Y}>30)$ smokers were positively associated with PCa, independently

of rs6983267, indicating probably different biological pathways in the process of carcinogenesis for these two factors regarding this type of cancer.

Smoke has an anti-estrogenic effect mainly caused by dosedependent elevated hepatic clearance of estrogens [13] and may also alter the metabolism of other sex steroids such as testosterone and adrenal hormones. Thereby, tobacco smoke may influence negatively the incidence of benign and malignant growth of the prostate gland [14] In adolescent male humans it also decreases the serum levels of folliclestimulating hormone (FSH) [15] while in male rats it has a direct toxic effect on Leydig cells reducing the production of testosterone [16,17].

The intermediate smokers $(\mathbf{P Y = 1 0 - 3 0})$ haven't been associated with the disease probably because this amount of inhaled smoke could provoke an inhibitory effect to hormonal pathways acting beneficially on the status of PCa development, without severe interference in DNA repair mechanisms [13-17]. Very light smokers $(\mathbf{P Y}<\mathbf{1 0})$ or nonsmokers were positively associated with prostate cancer, probably because of the existing effect of hormones in conjunction with other factors such as family history and rs6983267.

Heavy smokers (PY>30) were also positively associated with $\mathrm{PCa}$, probably because smoke has a multiple effect in enzymatic systems aggravating the carcinogenic process. In that case smoke is influencing positively $\mathrm{PCa}$ susceptibility in conjunction with all the other established factors. Tobacco smoke is one of the most common sources of cadmium (Cd) in the general population. At the cellular level, cadmium affects proliferation, differentiation and causes apoptosis. It has been classified as a carcinogen by the International Agency for Research on Cancer (IARC). Indirect effects of cadmium provoke generation of reactive oxygen species (ROS) and DNA damage. Cadmium modulates also specific gene expression and signal transduction and reduces activities of proteins involved in antioxidant defences [18].

Also, genetic polymorphisms in genes expressing phase I and II metabolic enzymes, in conjunction with smoke have been reported to enhance the PCa susceptibility. Constitutive, hormonal and cancer specific factors like benzo (a) pyrene included in smoke, affect the expression and induction of the phase I metabolic enzymes, CYP1A1 and CYP1A2, in prostate cells resulting to an increased metabolism of nicotine and therefore to an increased intake by the smokers and to an increased carcinogenic process [19]. In addition, some polymorphisms of phase II glutathione S-transferase enzymes (GSTP1, GSTM3, GSTM1) decrease the ability to detoxify carcinogen compounds found in cigarette smoke and modify PCa susceptibility [20-22]. Finally, the rapid arylamine $\mathrm{N}$-acetyltransferase 2 (NAT2) genotype which plays a major role in the metabolic activation of carcinogenic amines of smoke, has been correlated with the development of PCa risk [23,24].

Smoke also affects bone metabolism by different mechanisms. It induces proliferation and cytokine release in human osteoblasts and stimulates bone matrix turnover by increasing production of tissuetype plasminogen activator (tPA) and metalloproteinases (MMPs) $[25,26]$. These mechanisms tip the balance toward the resorption process and lead to bone loss, something that also characterise the PCa metastatic disease and partially explain the positive association between current smoking and fatal cancer of the prostate, in many prospective cohort studies [11]. All these observations converge in the smoking-linked higher risk of PCa development and morbidity of PCa patients who smoke.

In order to reach more safe and clear conclusions, further 
investigations should be performed analyzing the effect of different exposure to smoke in the various stages of $\mathrm{PCa}$ and the carcinogenic pathways in which smoke is involved.

In conclusion, our findings support the established model for PCa, of being a complex disease with genetic and environmental factors contributing to the carcinogenesis through different mechanisms.

\section{References}

1. Gronberg H (2003) Prostate cancer epidemiology. Lancet 361: 859-864.

2. Xu J, Dimitrov L, Chang BL, Adams TS, Turner AR, et al. (2005) A combined genomewide linkage scan of 1,233 families for prostate cancer-susceptibility genes conducted by the international consortium for prostate cancer genetics. Am J Hum Genet 77: 219-229.

3. Yeager M, Orr N, Hayes RB, Jacobs KB, Kraft P, et al. (2007) Genome-wide association study of prostate cancer identifies a second risk locus at $8 q 24$. Nat Genet 39: 645-649.

4. Haiman CA, Patterson N, Freedman ML, Myers SR, Pike MC, et al. (2007) Multiple regions within 8q24 independently affect risk for prostate cancer. Nat Genet 39: 638-644.

5. Zheng SL, Sun J, Cheng Y, Li G, Hsu FC, et al. (2007) Association between two unlinked loci at 8q24 and prostate cancer risk among European Americans. J Natl Cancer Inst 99: 1525-1533.

6. Zheng SL, Sun J, Wiklund F, Smith S, Stattin P, et al. (2008) Cumulative Association of Five Genetic Variants with Prostate Cancer. N Engl J Med 358: 910-919.

7. Yeager M, Xiao N, Hayes RB, Bouffard P, Desany B, et al. (2008) Comprehensive resequence analysis of a $136 \mathrm{~kb}$ region of human chromosome 8q24 associated with prostate and colon cancers. Hum Genet 124: 161-170.

8. Wokolorczyk D, Gliniewicz B, Sikorski A, Zlowocka E, Masojc B, et al. (2008) A range of cancers is associated with the rs6983267 marker on chromosome 8 . Cancer Res 68: 9982-9986.

9. Park SL, Chang SC, Cai L, Cordon-Cardo C, Ding BG, et al. (2008) Associations between variants of the 8q24 chromosome and nine smoking-related cancer sites. Cancer Epidemiol Biomarkers Prev 17: 3193-3202.

10. Mikhailidis DP, Ganotakis ES, Papadakis JA, Jeremy JY (1998) Smoking and urological disease. J R Soc Promot Health 118: 210-212.

11. Watters JL, Park Y, Hollenbeck A, Schatzkin A, Albanes D (2009) Cigarette smoking and prostate cancer in a prospective US cohort study. Cancer Epidemiol Biomarkers Prev 18: 2427-2435.

12. Papanikolopoulou A, Landt O, Ntoumas K, Bolomitis S, Tyritzis SI, et al. (2011) The multi-cancer marker, rs6983267, located at region 3 of chromosome 8q24 is associated with prostate cancer in Greek patients but does not contribute to the aggressiveness of the disease. Clin Chem Lab Med.

13. Mueck AO, Seeger H (2005) Smoking, estradiol metabolism and hormone replacement therapy. Curr Med Chem Cardiovasc Hematol Agents 3: 45-54.

14. Matzkin H, Soloway MS (1993) Cigarette smoking: a review of possible associations with benign prostatic hyperplasia and prostate cancer. Prostate 22: $277-290$.

15. Richthoff J, Elzanaty S, Rylander L, Hagmar L, Giwercman A (2008) Association between tobacco exposure and reproductive parameters in adolescent males. Int J Androl 31: 31-39.

16. Rajpurkar A, Li H, Dhabuwala CB (2000) Morphometric analysis of rat testis following chronic exposure to cigarette smoke. J Environ Pathol Toxicol Oncol 19: 363-368.

17. Zhan XS, Wu TP, Ouyang H, Liu XB, Cai HL (2009) Cigarette smoke affects sexual function of male rats. Zhonghua Nan Ke Xue 15: 788-791.

18. Bertin G, Averbeck D (2006) Cadmium: cellular effects, modifications of biomolecules, modulation of DNA repair and genotoxic consequences (a review). Biochimie 88: 1549-1559.

19. Sterling KM Jr, Cutroneo KR (2004) Constitutive and inducible expression of cytochromes P4501A (CYP1A1 and CYP1A2) in normal prostate and prostate cancer cells. J Cell Biochem 91: 423-429.

20. Mao GE, Morris G, Lu QY, Cao W, Reuter VE, et al. (2004) Glutathione S-transferase P1 lle105Val polymorphism, cigarette smoking and prostate cancer. Cancer Detect Prev 28: 368-374.

21. Kesarwani P, Singh R, Mittal RD (2009) Association of GSTM3 intron 6 varian with cigarette smoking, tobacco chewing and alcohol as modifier factors for prostate cancer risk. Arch Toxicol 83: 351-356.

22. Lavender NA, Benford ML, VanCleave TT, Brock GN, Kittles RA, et al. (2009) Examination of polymorphic glutathione S-transferase (GST) genes, tobacco smoking and prostate cancer risk among men of African descent: a casecontrol study. BMC Cancer 9: 397.

23. Wang CY, Jones RF, Debiec-Rychter M, Soos G, Haas GP (2002) Correlation of the genotypes for $\mathrm{N}$-acetyltransferases 1 and 2 with double bladder and prostate cancers in a case-comparison study. Anticancer Res 22: 3529-3535.

24. Costa S, Pinto D, Morais A, Vasconcelos A, Oliveira J, et al. (2005) Acetylation genotype and the genetic susceptibility to prostate cancer in a southern European population. Prostate 64: 246-252.

25. Kamer AR, El-Ghorab N, Marzec N, Margarone JE 3rd, Dziak R (2006) Nicotine induced proliferation and cytokine release in osteoblastic cells. Int $\mathrm{J}$ Mol Med 17: $121-127$

26. Katono T, Kawato T, Tanabe N, Suzuki N, Yamanaka K, et al. (2006) Nicotine treatment induces expression of matrix metalloproteinases in human osteoblastic Saos-2 cells. Acta Biochim Biophys Sin (Shanghai) 38: 874-882. 